\title{
Gravet, C., Lievois, K. (Eds.) (2021). Vous avez dit littérature belge francophone ? Le défi de la traduction. Peter Lang. (pp. 436)
}

Ce volume cadre dans l'intérêt croissant pour une problématique longtemps négligée, à savoir I'histoire de la traduction, en particulier des littératures dites 'mineures', comme, en l'occurrence, la littérature belge francophone. Complexe comme l'est le pays avec sa culture trilingue en périphérie des grands voisins, cette histoire de la traduction belge, qui n'en est qu'au stade de l'enfance, a récemment été comparée à un dilemme de Buridan (D'hulst, 2019) : tout comme l'âne allégorique d'Aristote, qui meurt de faim faute de choisir entre deux options à valeur égale, l'historiographe de la traduction se voit placé.e devant une alternative. Soit on choisit d'étudier les intraductions présentes dans le pays, mais c'est aussi s'inscrire dans le prolongement des histoires nationales auxquelles on cherche pourtant à faire contrepoids ; soit on se concentre - comme c'est le cas ici - sur les extraductions d'œuvres écrites dans la (ou les) langue(s) concernée(s), dans une visée transnationale et solidaire des débats sur la World Literature, ce qui comporte cependant le risque de voir l'ancrage national (et régional) des œuvres se diluer dans le grand concert des langues. Or quelle que soit l'option que l'on choisisse, d'importantes questions de méthode demeurent, voire se posent avec plus d'insistance dans le cas belge - on connaît le rapport difficile des auteurs belges, francophones et autres, avec leur ancrage 'national' et le rôle que jouent à cet égard les aléas d'une vie littéraire orientée sur le grand voisin qui décide non seulement du succès, mais du succès international des œuvres. Pour être traduits, les auteurs francophones belges doivent d'abord, en règle générale, se faire accepter à Paris ; lorsque traduits, ils sont (donc) souvent perçus à l'étranger comme appartenant au grand ensemble français. Aussi la littérature belge peine-t-elle à se donner une visibilité internationale, d'autant, insistent les éditrices dans leur introduction (p. 11), qu'une véritable politique de traduction fait défaut.

Comment, donc, mettre sur le métier le " projet d'envergure » qui consiste à décrire " toutes les conditions de production des traductions " (p. 17), ainsi que leur réception dans les cultures d'accueil ? Convient-il de se concentrer sur une seule langue et de produire, donc, plusieurs histoires de la traduction belge (francophone, néerlandophone, germanophone) en parallèle, ou faut-il tenter une histoire globale, plurilingue, de la traduction belge ? se concentrer sur les seules traductions d'œuvres à l'étranger ou tenir compte, aussi, des intraductions, sans oublier par ailleurs les traductions intrabelges ? Comment inscrire, dans une telle histoire, l'évolution de la question linguistique en Belgique : en tant que donnée structurante ou comme symptôme d'une réalité socio-politique, puis littéraire, dont les retombées sur la traduction n'ont pas toujours été les mêmes ? Quant au corpus, quels auteurs traduits étudier : doivent-ils être de nationalité belge ou avoir (eu) un 'ancrage' en Belgique ? Que faire d'auteurs d'avant 1830, traduits après, comme Jean-Joseph de Ligne ? Et quels genres, notamment paralittéraires, inclure : science-fiction, BD, roman-photo, chanson ? Faute de consensus, qui est loin d'être établi, sur ces questions, par où commencer, d'autant, soulignent les éditrices, que les sources bibliographiques fiables sur les traductions manquent et que les fonds d'archives réunissant traductions et archives des traducteurs font défaut.

Face à ce dilemme, Catherine Gravet et Katrien Lievois ont pris le parti - et pour cela, elles méritent éloge - de ne pas se comporter comme l'âne de Buridan, mais de commencer à documenter la « visibilité internationale » (p. 11) des œuvres d'auteurs belges francophones. L'accent est donc mis sur les extraductions, en particulier sur l'agencement de médiateurs culturels étrangers souvent inconnus en Belgique, dans des langues moins 'centrales' comme 
le grec, le roumain, le turc, le bulgare (ni l'anglais ni le néerlandais ne figurent parmi les langues de traduction étudiées).

A cet égard, ce livre fait complément à plusieurs volumes parus auparavant. Entamée avec les portraits de Traducteurs et traductrices belges (Gravet, 2013), cette approche documentaire a été poursuivie par l'ouvrage Traduire la littérature belge francophone (Costa \& Gravet, 2016) et par le colloque "La traduction de la littérature belge francophone » organisé par I'Université de Mons, en collaboration avec l'Université d'Anvers, l'Académie Royale de Langue et de Littérature Françaises de Belgique et les Archives et Musée de la Littérature, les 13 et 14 décembre 2018. De ce colloque sont issues deux publications : un numéro spécial de la revue de traductologie Parallèles (Gravet \& Lievois, 2020) - voir ici même le compte rendu par Francis Mus - et le présent volume dans lequel les approches sont plus variées, combinant traductologie et études littéraires, imagologie, voire "cantologie ". Là où le numéro de Parallèles creuse la question en ralliant les cas étudiés à des questionnements méthodologiques en traductologie, notamment dans l'excellente introduction des éditrices, le présent volume répond plutôt à une logique de la largeur, sans proposer de « théorie à proprement dire » (p. 17).

Le volume contient seize contributions, distribuées sur quatre parties. Dans une première partie intitulée " traduction \& création », il est question des traductions grecques de Maeterlinck (Maria Baïraktari) ; de deux traductions bulgares, précoce et récente, " relevante " et "défaillante ", de Bruges-la-Morte de Rodenbach (Irena Kristeva) ; de la question de savoir s'il faut, et à quel prix, traduire Brel (Stéphane Hirschi) ; et des traductions espagnole de Fuir (Juan Miguel Dothas) et italienne de Faire l'amour (Thea Rimini) de Toussaint. La seconde partie, "travail \& archives des traducteurs ", ouvre sur le témoignage d'une expérience didactique de la traduction à partir d'une nouvelle de Marie Delcourt (Béatrice Costa) ; pour passer à la correspondance de Yourcenar avec ses traducteurs (Mireille Brémond) ; à une auto-analyse de la traductrice roumaine d'CEdipe sur la route d'Henry Bauchau (Rodica LascuPop) ; enfin à la traduction italienne de Musculatures de l'écrivaine culturiste Nathalie Gassel (Claudio Grimaldi). Dans la partie " traduction \& réfraction », ensuite, on trouve une analyse du paysage dans la traduction italienne d'Un mâle de Lemonnier (Maria Giovanna Petrillo) ; une exploration du paysage sémiotique de la ville d'Istanbul dans Les clients d'Avrenos de Simenon (Sündüz Öztürk Kasar) ; une analyse des traductions et de la réception espagnoles de l'CEuvre au noir de Yourcenar (André Bénit) ; de la traduction allemande du roman à succès Passeur de lumière de l'écrivain-artiste Bernard Tirtiaux (Marie Fortunati) ; enfin des traductions allemandes des romans d'Amélie Nothomb (Anja van de Pol-Tegge). Le volume conclut sur deux précieux " inventaires », d'une part des 199 traductions grecques d'auteurs belges francophones (Fanny Sofronidou) ; d'autre part de la présence d'auteurs belges dans la prestigieuse anthologie mexicaine de littérature francophone par Laura López Morales (Thomas Barège).

Si ce volume renferme, ainsi, une documentation précieuse et variée, son approche ne va pas sans inconvénients. D'abord, mais c'est sans doute inévitable, on rencontre avant tout les usual suspects (même si plusieurs contributions sont, comme on l'a vu, consacrées à des noms moins connus) : Maeterlinck, Rodenbach, Lemonnier, Simenon, Nothomb, auxquels s'ajoutent Brel et, comme il est de règle, Yourcenar et Toussaint, auteurs-phares dont on peut cependant se demander dans quelle mesure ils sont véritablement belges. Puis, les contributions se penchent toutes sur la traduction vers une seule langue, de sorte que la vue d'ensemble demeure fragmentée. Ensuite, les approches sont variées, allant de l'étude de cas d'un 
texte traduit, à travers des considérations cantologiques et l'analyse de correspondance, à la description de corpus d'un auteur ou des auteurs belges traduits vers une langue donnée. En résulte une logique d'ensemble qui peut paraître disparate. Enfin, les éditrices du volume ont ratissé large en donnant la parole à la grande majorité des participants au colloque de Mons, soit dans le numéro spécial de Parallèles (qui regroupe dix articles, dont sept remontent audit colloque), soit dans le présent volume (dont seules trois contributions n'ont pas été présentées à Mons). Si l'on comprend ce choix sans doute inspiré par la volonté de rassembler un maximum de données, force est de constater que les analyses présentées manquent parfois de rigueur ou de profondeur. Aussi convient-il de considérer ces deux publications comme un diptyque dont l'intérêt consiste, pour l'une, à ouvrir le débat et à poser les premiers jalons méthodologiques et, pour l'autre, à réunir des données variées et précieuses. C'est avant tout, donc, sur le plan de la documentation, indispensable à l'histoire en devenir de la littérature belge (francophone) en traduction, que ce volume mérite sa place.

\section{Références}

Costa, B. \& C. Gravet (éds). (2017). Traduire la littérature belge francophone. Itinéraires des œuvres et des personnes. Mons : Université de Mons.

D'hulst, L. (2019). Le dilemme de Buridan : Une histoire de la traduction belge est-elle possible? Chronotopos 1/2019, 65-80.

Gravet, C. (éd.). (2013). Traductrices et traducteurs belges. Mons : Université de Mons.

Gravet, C. \& K. Lievois. (Eds). (2020). La littérature francophone belge en traduction. Parallèles 32 (1).

Kris Peeters

Université d'Anvers

Kris.peeters@uantwerpen.be 